\title{
Alcoholic Liver Disease And Immune Response
}

\author{
Khem B. Adhikari ${ }^{\dagger, *}$ and Oren Tirosh \\ Institute of Biochemistry, Food Science and Nutrition, Robert H. Smith Faculty of Agriculture, Food and Environment, \\ The Hebrew University of Jerusalem, Israel \\ ${ }^{\dagger}$ Current affiliation: Department of Agroecology, Aarhus University, Denmark
}

\begin{abstract}
The alcoholic liver disease (ALD) continues to be a major health problem worldwide with respect to both morbidity and mortality. It is usually an outcome of excessive alcohol consumptions but can be worse by poor diet and genetic factors. This review aims to update the recent conditions of the epidemiology, pathogenesis, immune response and therapies to ALD. The new insights into the pathogenesis of ALD include the key roles of the excess production of cytokines, reactive oxygen species (ROS) and the shortage of protective mediators, including adiponectin. As the liver injury progresses, the immune system fails to counteract proinflammatory cytokines and ROS which further cause oxidative liver injury. By inducing mitochondrial alterations, oxidative stress promotes hepatocyte necrosis, release of pro-fibrotic cytokines and activation of collagen gene expression in hepatic stellate cells contributing to alcohol induced sensitization of hepatocyte to pro-apoptotic action of TNF- $\alpha$. ALD can progress to cirrhosis, hepatocellular carcinoma, and liver failure. Regulation of perioxisome proliferator activated receptor (PPAR) and sterol regulatory element binding protein (SREBP) accompanied with lifestyle modifications are currently in practice for alleviating ALD.
\end{abstract}

Keywords: ALD, cytokines, ethanol, immune response, oxidative stress.

\section{INTRODUCTION}

Alcoholic liver disease (ALD) is a common consequence of long term alcohol abuse [1] and represents a major cause of mortality and morbidity worldwide [2-4]. ALD encompasses a broad spectrum of morphological features ranging from simple steatosis with minimal injury to more advanced stage liver injury, including alcoholic steatohepatitis, alcoholic fibrosis and alcoholic cirrhosis increasing liver stiffness $[1,2,5]$. The risk of steatosis, inflammation and fibrosis are more common in alcoholics [6] and increases with time and the amount of ethanol consumed [7]. This review has discussed in detail the pathogenesis, progression, immune response, and treatment practices for the attenuation of ALD.

\section{CLINICAL ASPECTS OF ALD}

Fatty liver, the most common syndrome of ALD, is characterized by the excessive accumulation of fat inside hepatocytes [8]. Excessive fat accumulation in the hepatocytes is the most common and earliest response of the liver to chronic alcohol consumption [9]. In some cases, alcohol abuse can lead to inflammation of fatty liver called alcoholic steatohepatitis. Morphological criteria of steatohepatitis are steatosis, ballooning of hepatocytes, pericellular fibrosis and inflammation [10]. In an animal model of ALD, rats exposed 4 weeks to alcohol exhibited a significant increase in liver to body weight ratio, serum alanine aminotransferase (ALT) levels and hepatic TNF- $\alpha$ compared to control group [9]. Ithas been found that the levels of alkaline phosphate, ALT,

*Address correspondence to this author at the Department of Agroecology, Aarhus University, Denmark; Tel: +45 87158139; Fax: +45 87156082;

E-mail: khem-bah.adhikari@mail.huji.ac.il protein and globulin were significantly increased in alcoholic males compared to control subjects [11]. The AST (Aspartate aminotransferase) /ALT ratio is significantly higher in ALD patients sometimes even greater than two [8]. People who continue abusing alcohol may develop more serious liver damages like fibrosis, cirrhosis, and even liver failure $[1,2]$.

\section{MECHANISMS AND IMMUNE RESPONSE}

There are multiple mechanisms for the development and progression of ALD (Fig. 1) and many of these mechanisms interact to each other [3]. The role of liver for ethanol metabolism, hepatocyte toxicity, production of cytokines, and contribution of genetic makeup and dietary history is discussed.

\section{ETHANOL METABOLISM AND ROLE OF ACETAL- DEHYDE}

The liver breaks down alcohol so that it can be eliminated from our body. However, when it is over consumed than the liver can process, the resulting imbalance can injure the liver by interfering its normal breakdown of proteins, fats, and carbohydrates [12]. ALD has a complex pathogenesis, in which acetaldehyde; the major ethanol metabolite plays a central role [13]. Alcohol is primarily metabolized by the successive oxidative activities of alcohol dehydrogenase $(\mathrm{ADH})$ and acetaldehyde dehydrogenase [(ALDH), Fig. 2] $[14,15]$. Ethanol is metabolized mainly in the hepatocytes in three different sites (Fig. 3): cytosol, endoplasmic reticulum and peroxisome while the acetaldehyde is metabolized in mitochondria [16]. The main pathway involves cytoplasmic ADH which catalyzes the oxidation of ethanol to acetalde- 


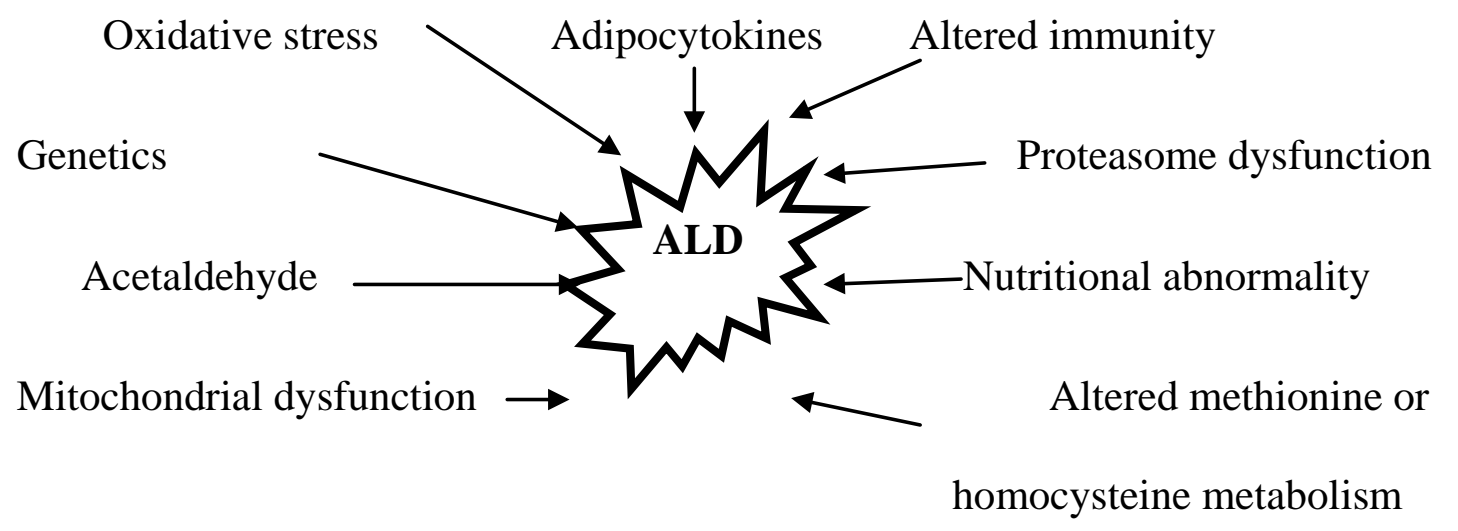

Fig. (1). Different mechanisms for the development of ALD.

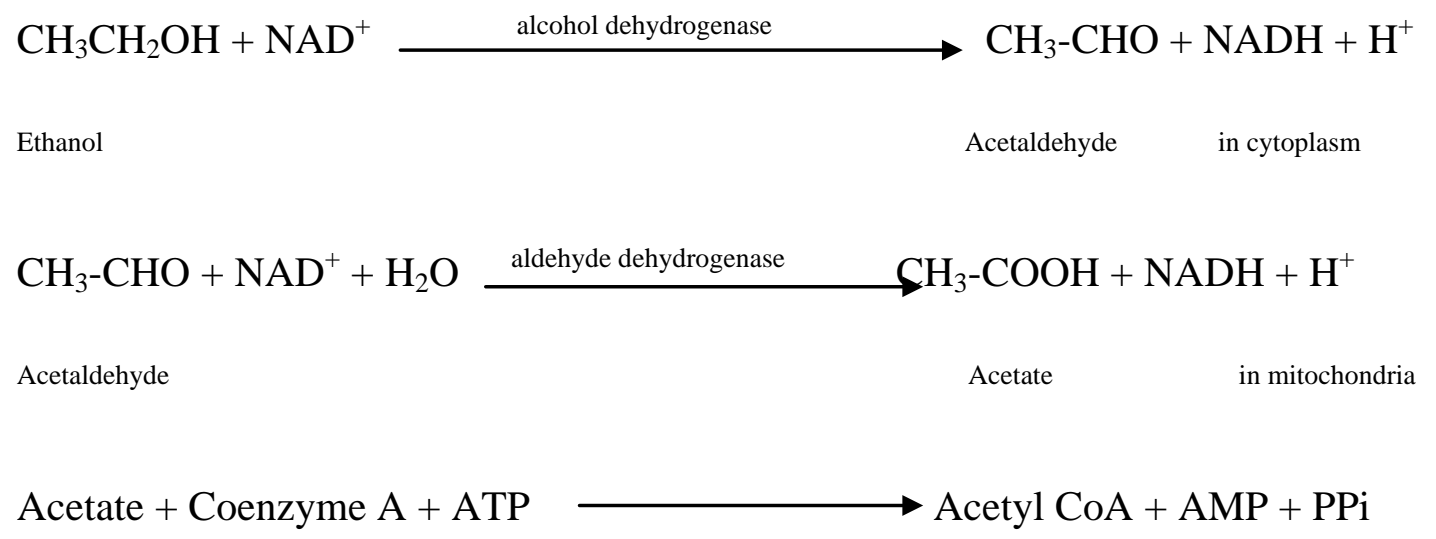

Fig. (2). Ethanol metabolism.

hyde then oxidized to acetate by the mitochondrial ALDH [13]. The acetaldehyde enhances the formation of (reactive oxygen species) ROS [17]. Because ethanol-inducible CYP2E1 can generate ROS during its catalytic circle; and its levels are elevated by chronic ethanol consumption, CYP2E1 has been suggested to be a major contributor to ethanolinduced oxidative stress and to ethanol-induced liver injury [18]. Ethanol induces CYP2E1 which metabolizes and activates many toxicological substrates, including ethanol, to more reactive toxic products [18].

Severely toxic manifestations are produced by an accessory inducible pathway, the microsomal ethanol-oxidizing system (MEOS) in endoplasmic reticulum involving an ethanol-inducible CYP2E1 in which the oxidation of ethanol to acetaldehyde and acetate also leads to generation of ROS [hydroxyethyl free radicals, hydrogen peroxides $\left(\mathrm{H}_{2} \mathrm{O}_{2}\right)$ and super oxide anion $\left(\mathrm{O}_{2}^{-}\right)$] [13]. High reduced nicotinamide adenine dinucleotide (NADH) is produced due to alcohol metabolism leading to high $\mathrm{NADH} / \mathrm{NAD}^{+}$ratio which overrides the cell's ability to maintain normal redox state [15].

In a study CYP2E1 activity was increased about 4 fold and TBARS levels, a marker of oxidative stress was increased about 9 fold in alcohol fed wild type mice with increased hepatic accumulation of triglyceride indicating that CYP2E1-derived oxidative stress may inhibit oxidation of fatty acids by preventing up-regulation of perioxisome proliferator activated receptor (PPAR)- $\alpha$ [19]. They also found that PPAR- $\alpha$-regulated acyl CoA oxidase was decreased more than 2 fold after ethanol feeding in wild type mice but was not decreased in the CYP2E1 knockout mice. Similarly administration of CYP2E1 to ethanol fed CYP2E1 knockout mice restored steatosis, and use of chlormethiazole to inhibit CYP2E1, blocked ethanol-induced steatosis in wild type mice indicating that CYP2E1 play a role in ALD [19].

Chronic ethanol consumption increases fatty acid synthesis by inducing the expression of lipogenic enzymes which are regulated by transcription factor, sterol regulatory element binding protein (SREBP) [8]. Chronic ethanol consumption significantly inhibits mitochondrial ALDH activity while the rate of ethanol oxidation to acetaldehyde is even enhanced, resulting in striking increase in tissue and plasma levels of acetaldehyde which results in metabolic disturbances, such as hyperlactacidemia, acidosis, hyperglycemia, hyperuricemia and fatty liver [13].

\section{TUMOR NECROSIS FACTOR- $\alpha$}

Under normal condition, hepatocytes are resistant to TNF- $\alpha$ induced killing but there is a mechanism by which chronic alcohol exposure sensitizes to TNF- $\alpha$ hepatotoxicity which was shown in animal model of ALD [20]. The production of TNF- $\alpha$ is one of the earliest events in liver injury, triggering the production of other cytokines that together recruit inflammatory cells, kill hepatocytes, and even initiate healing response that includes fibrogenesis (Fig. 4) [21]. It has been reported that in both chronic alcohol-fed rats and heavy drinkers, the elevation of Immunoglobulin G ( $\operatorname{IgG}$ ) 


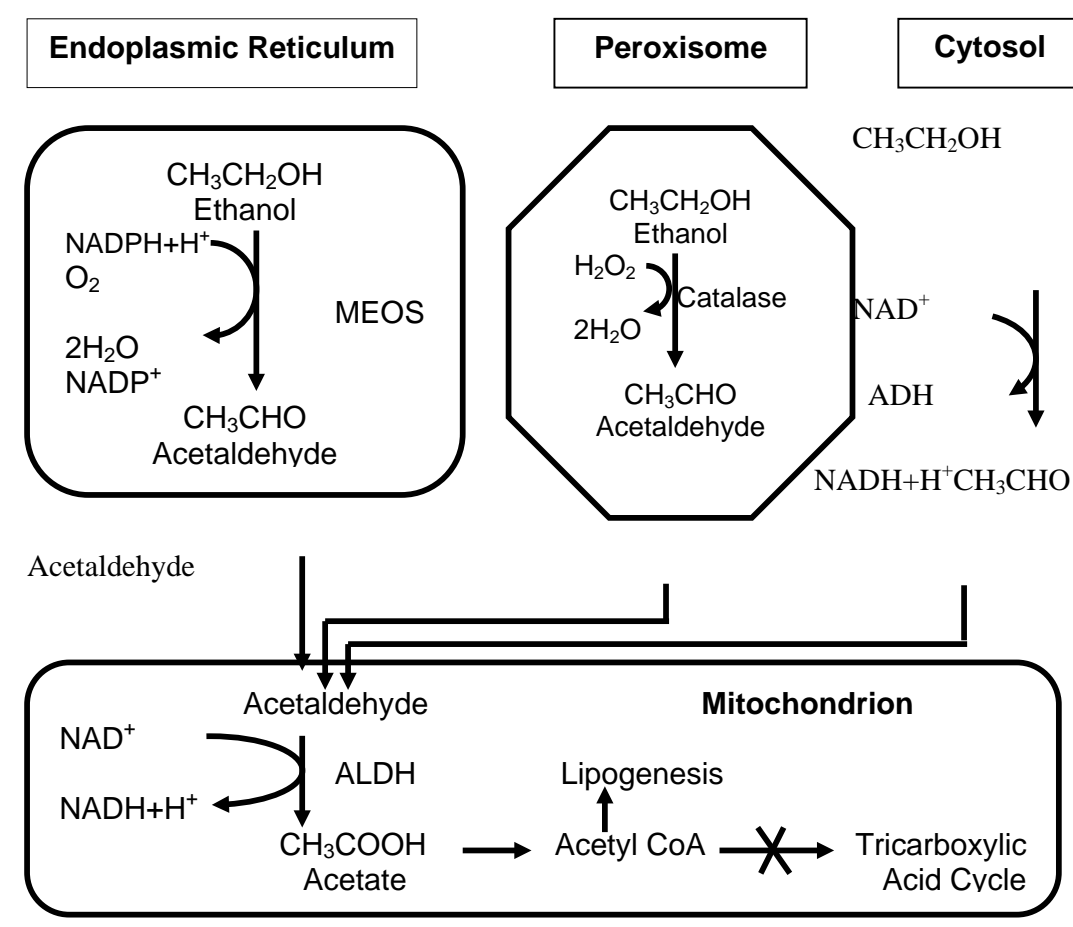

Fig. (3). Ethanol metabolism in hepatocytes. These mechanisms are potentially involved in oxidative stress production. Ethanol is metabolized in acetaldehyde and then transformed into acetate. High NADH and low NAD ${ }^{+}$impair the tricarboxylic acid cycle so that acetyl CoA diverts into lipogenic pathway.

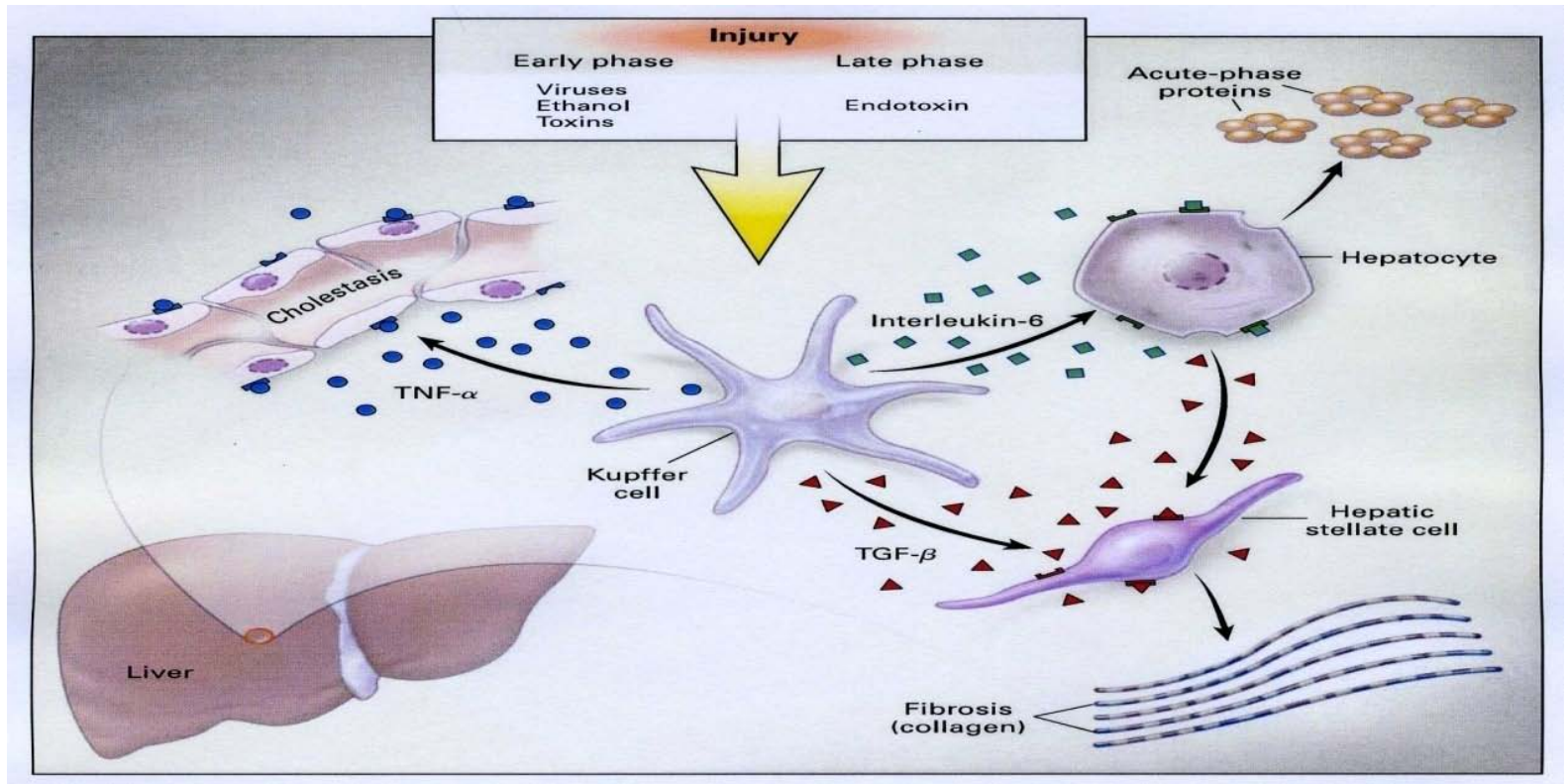

Fig. (4). Pathologic relevance of cytokines in chronic liver disease [21] (cited with permission). Most types of cells in the liver either synthesize or respond to cytokines. In early phase, ethanol, and in the late phase endotoxin stimulates cytokine production. Proinflammatory cytokine TNF- $\alpha$ involves in cholestasis, anti-inflammatory cytokine interleukin (IL)-6 involves in synthesis of acute phase protein. Transforming growth factor (TGF)- $\beta$ released by hepatocytes and the kupffer cells involve in fibrosis.

against lipid peroxidation-derived antigens is associated with TNF- $\alpha$ production and the severity of liver inflammation [7].

Oxidative mitochondrial damage is one of the mechanisms of hepatocyte apoptosis and/or necrosis in ALD [2]. The TNF- $\alpha$ activates caspase- 8 , a protease, in the cytoplasm which cleaves protein Bid responding to release of cyto- chrome c oxidase from the mitochondria [21]. Cytochrome c oxidase activates caspase-3 and causes apoptosis [21]. Similarly TNF- $\alpha$ induces sphingomylinase which increases ceramide, an inhibitor of the activity of mitochondrial electron transport chain, leading to increased mitochondrial production of ROS which in turn promote lipid peroxidation and cellular necrosis [21]. 
TNF- $\alpha$ activates a stress related protein kinase cascade with Jun N-terminal kinase kinase 1 (SEK1) and Jun Nterminal kinase that phosphorylate proto-oncogene C-Jun, helping mitogens to promote proliferation [21]. They also reported that ROS also contribute to the activation of transcription factors (nuclear factor-kB and adaptor protein 1) that are necessary for the synthesis of cell survival factors (MnSOD, Bfl-1, Bcl- $\mathrm{x}_{\mathrm{L}}$ ) which protect mitochondria. NF-kB activation induces the transcription of antiapoptotic proteins which prevents from TNFa induced cell death [20]. However, the immune responses towards antigens originating from the reactions between lipid peroxidation products and hepatic proteins also contribute to continue chronic hepatic inflammation in ALD [2]. In patients with progressive liver disease, the balance between proinflammatory and antiinflammatory cytokines may be shifted toward the proinflammatory axis, thus, the counteracting anti-inflammatory cytokines are unable to control inflammation and fibrosis [21]. Inflammation and necrosis in the liver trigger fibrosis as a part of the wound healing and scarring response [8].

\section{INTERLEUKINS}

Several other pro- or anti- inflammatory cytokines are produced by liver in response to alcoholic liver injury. Interleukin (IL) - 6, an anti-inflammatory cytokine involves in synthesis of acute phase protein (Fig. 4) [21]. IL - 10, another anti-inflammatory cytokine is found to be involved in inflammatory response instead it was shown to be resistant to ethanol induced steatosis and hepatocyte damage [22]. That was made possible by elevation of IL - 6, up-regulation of fatty acid oxidation-associated genes and down-regulation of lipogenic genes in IL - 10 knockout mice. Likewise, IL - 22, another member of IL -10 family of cytokines, holds the ability to keep liver homeostasis and tissue repair. It triggers the activation of signal transducer and activator of transcription 3 (STAT3) signaling pathway including others that has protective effect against ethanol-induced liver injury [23].

\section{ADIPONECTIN}

Adiponectin is an anti-inflammatory adipokine which reduces the liver injury by inhibiting stellate cell activation [24]. It is reported that gene expression of adiponectin is down regulated by high homocysteine levels in alcoholic patients so it enhances the pathogenesis of ALD [9].

\section{OXIDATIVE AND NITROSATIVE STRESS}

Above a certain limit, ROS cannot be efficiently removed by antioxidant system and become important mediators of liver damage [16]. Oxidative stress occurs when high cellular levels of ROS overwhelm the antioxidants [25]. Oxidative and nitrosative stress play an important role in the pathogenesis of ALD [18, 25, 26]. Oxidative stress associated alcoholic toxicity is mainly caused by ROS, generated by mitochondrial respiratory chain, the ethanol inducible cytochrome CYP2E1 of hepatocytes and the NADPH oxidase of Kupffer cells and liver infiltrating granulocytes [2, 14] together with impaired antioxidant defense system [10]. NADPH oxidase complex, which plays an important role in ROS production, is over expressed in hepatocytes, hepatic stellate cells, and kupffer cells in the liver of alcoholic patient [16].

It is reported that ethanol-induced CYP2E1 also makes liver more vulnerable to LPS-endotoxin injury [19]. It is reported that CYP2E1 yields increased acetaldehyde generation, antibody production, enzyme inactivation, decreased DNA repair, impaired oxygen utilization, glutathione (GSH) depletion, free radical mediated toxicity, lipid peroxidation, activation of xenobiotics to toxic metabolites, and increased collagen synthesis [13]. CYP2E1 itself is also an effective generator of ROS such as $\mathrm{O}_{2}^{-}$and $\mathrm{H}_{2} \mathrm{O}_{2}$; and iron catalyzes CYP2E1 to produce powerful oxidant, hydroxyethyl free radical [18]. Free radicals are capable of initiating peroxidation of the PUFA chains of phospholipids and lipoprotein in the cell membrane which cause cell necrosis and megamitochondria [27]. In an animal model of ALD hydroxyethyl free radicals were correlated with both stimulation of lipid peroxidation and liver injury [28]. They also found a positive correlation $(\mathrm{r}=0.732 ; \mathrm{P}<0.002)$ between CYP2E1 and hydroxyethyl radicals in ethanol fed rats. Furthermore, a decrease of CYP2E1 content in the liver of alcohol fed rats treated with diallylsulfide (DAS) and phenylethyl isothiocyanate (PIC), both CYP2E1 inhibitors, decreased hydroxyethyl free radical generation, peroxidative damage (low level of lipid peroxidation product-malonildialdehyde) and hepatotoxicity, suggesting that CYP2E1 and ethanol derived free radicals might play a role in onset of liver injury [28]. So there is a close link between the induction of CYP2E1 by ethanol, the formation of hydroxyethyl radicals and the stimulation of lipid peroxidation [28].

In animal model of ALD, thiobarbituric acid reactive substances [(TBARS), end products of lipid peroxidation with malondialdehyde (MDA) as a main component] level was increased; and superoxide dismutase [(SOD), a powerful enzymatic cellular antioxidant] activity and GSH content were decreased [29]. GSH is a strong protector against ROS injury but its level was decreased by acetaldehyde, accumulating ROS [17]. Intake of alcohol results in excessive generation of free radicals, which alter the biomembranes and cause damage [26]. Oxidative damage induced by ethanol contributes to the pathogenesis of alcoholic hepatotoxicity in many ways, one of them is it triggers the release of profibrotic cytokines (TGF- $\beta$ ) and activates hepatic stellate cells to produce collagen and progress into liver fibrosis (Fig. 4) $[2,17,30]$.

\section{MITOCHONDRIAL DYSFUNCTION}

One of the earliest effects of chronic ethanol consumption on liver is an alteration in mitochondrial structure especially with enlarged and misshapen organelle and disrupted cristae so that there is a decreased rate of adenosine triphosphate (ATP) synthesis via oxidative phosphorylation system [31]. They have also reported that excess NADH is generated during ethanol oxidation which is shuttled into mitochondria from cytosol through activated malate-aspartate shuttle and as a consequence of this increased flux of electrons, the mitochondrial electron transport chain components will be more reduced, this in turn will facilitate increased transfer of electrons to molecular oxygen to generate super oxide anion $\left(\mathrm{O}_{2}^{-}\right)$. So the concentration of mitochondrial 
ROS can be elevated not only due to increased NADH flux as a result of ethanol oxidation, but also due to the alterations in electron transport chain complex I and III by chronic ethanol exposure [31]. Oxidative stress, by inducing mitochondrial alterations, promotes hepatocyte necrosis and contributes alcohol-induced sensitization of hepatocyte to proapoptotic action of TNF- $\alpha[2,30]$.

\section{ABNORMAL METHIONINE METABOLISM}

Abnormal methionine metabolism is one of the major metabolic abnormalities in ALD, characterized by decreased hepatic S-adenosyl methionine (SAMe), increased hepatic Sadinosylhomocysteine (SAH), decreased SAMe/SAH ratio, and hyperhomocysteinemia $[9,20]$. There is impaired conversion of methionine to s-SAMe because of decreased hepatic methionine adenosyltransferase activity [32, 33]. However, there is an increased level of homocysteine which sensitize TNF hepatotoxicity by down regulation of the gene expression of adiponectin in the adipose tissue [9, 32]. GSH is a cellular antioxidant consisting of glycine, cysteine, and glutamic acid residue [34]. Hepatic SAMe depletion due to chronic alcohol feeding can lead to membrane dysfunction caused by decreased transmethylation, depletion of polyamines, and inability to replete GSH $[14,34]$. Disruption of hepatic transmethylation pathway is associated with significant increases in hepatic TNF- $\alpha$ levels, capase- 8 (protease enzyme involved in apoptosis) activity and cell death in animal model of ALD $[9,20]$.

\section{INTESTINAL PERMEABILITY}

Alcohol disrupts the intestinal barrier function which leads to gut permeability across the epithelial cell layer allowing substances such as LPS to enter the circulation causing endotoxemia [14] which is strong inducer of proinflammatory cytokines such as TNF- $\alpha$ [35]. Larger molecules of polyethylene glycol (PEG) were recovered in about twice as high concentration in the urine of ALD patients in comparison to healthy patients ingested with PEG with different molecular masses indicating that alcohol impairs intestinal barrier casing significant increase in intestinal permeability to macromolecules [36]. It has been reported that kupffer cell activation by gut-derived endotoxins (LPS) release TNF- $\alpha$ and $\mathrm{O}_{2}^{-}$that result in an inflammatory response $[37,38]$.

\section{GENETIC FACTORS}

Genetic factors also influence the individual susceptibility for alcoholic liver disease [37]. Activated kupffer cellinduced proinflammatory cytokines are regulated by the CD14 endotoxin receptor gene [37]. They also reported that a $\mathrm{C} \rightarrow \mathrm{T}(-159)$ polymorphism in the promoter region of the CD14 gene was found to increase CD14 expression. Advanced ALD was much more frequent among the individuals possessing the T-allele at the -159 position of the promoter region of the CD14 gene [37]. Study based on forensically autopsied Finnish men suggested that carriers of $\mathrm{T}$ allele in the promoter region of CD14 receptor gene have a 3- to 4fold risk of developing alcoholic cirrhosis [37].

The analysis of gene expression by microarray found that upregulation of genes like Kupffer cell receptor (Kclr), ATP citrate lyase (Acly), cytochrome P450 7a1 (CYP7a1), fatty acid binding protein 1 (Fabp1), fatty acid synthase (Fasn), Sterol carrier protein 2 (Acp2), etc [39] and downregulation of genes involved in electron transport, oxidoreductase activity, etc. in the liver of ethanol fed female rats which made them more vulnerable to ALD than the male rats. Alcohol toxicity increases with age because of increased organ susceptibility caused by decrease in ethanol metabolizing enzyme activities, decrease in microsomal and mitochondrial functions, metabolic syndrome, and use of multiple drugs [40].

\section{POOR NUTRITION}

Poor dietary habits also may have contributed to oxidative stress in the patients because they consume relatively lower amount of antioxidant vitamins compared to control subjects [41]. They also found an association of reduced dietary vitamin A intake with the presence and severity of liver disease. Alcoholics have always been considered to be malnourished due to exchange of food calories [3, 38] which hastens the development of alcoholic liver injuries [34]. They also reported that alcoholic patients are often deficient in dietary antioxidants (vitamin $\mathrm{C}, \mathrm{E}$ ) as a result of malnutrition.

\section{IMMUNE REACTION}

Hydroxyethyl free radicals and lipid peroxidation derived antigens promote the development of both humoral and cellular immune responses against liver proteins in the hepatocytes undergoing ethanol-induced oxidative stress [7, 30]. This will again be an additional mechanism in the pathogenesis of ALD, as they contribute to the chronic inflammatory process [30]. The immune system is involved in the development and progression of ALD which was evidenced by the detection of circulating autoantibodies, hepatic infiltration of immune cells and the detection of hepatic aldehyde modified proteins in patients with ALD [38]. Similarly, elevation of AST and ALT, IL-10, IFN $\gamma$, and reduced activities of IL-2 and IL-4 in mice serum due to ethanol exposure indicated that oxidative stress and immunomodulatory activities were interdependent and associated with ethanol induced liver damage [29]. Ethanol affects many functions of the innate immune system [38] and the cell types involved in this early immune response include: macrophage, neutrophils, and natural killer cells.

\section{TREATMENT}

The principles of treating ALD are: alcohol withdrawal, nutritional support, and treating alcoholic cirrhosis and its complications (Table 1) [1]. Liver transplantation is lifesaving approach in patients with end stage liver disease [1, 4].

\section{ALCOHOL WITHDRAWAL}

Fatty liver is common to most adults who drink regularly and is reversible upon cessation of alcohol intake [34, 38].

\section{NUTRITIONAL SUPPORT}

Patients with ALD need sound nutritional support. On the basis of alcohol withdrawal, patients should take high pro- 
Table 1. Potentially Beneficial Treatments in Studies in Animals and Clinical Trials [13, 17, 21]

\begin{tabular}{|l|l|}
\hline \multicolumn{1}{|c|}{ Therapy } & \multicolumn{1}{c|}{ Purpose } \\
\hline \hline Antibiotics, Lactobacillus & Prevent gut-derived endotoxins \\
\hline Anti-TNF antibodies, TNF-receptor antagonists & Inhibit TNF- $\alpha$ activity \\
\hline Glucocorticoids & Inhibit inflammation \\
\hline $\begin{array}{l}\text { Glutathione prodrugs (S-adinosylmethionine, betaine, choline), Vitamin E, } \\
\text { Silymarin, propylthiouracil, Raloxifene }\end{array}$ & Decrease production of ROS \\
\hline Ursodiol, gemfibrozil & Reduce liver lipids \\
\hline Leptin, thiozolidinediones & Improves insulin sensitivity \\
\hline N-acetylcysteine, Adenosyl-L-methionine & Increase Glutathione \\
\hline
\end{tabular}

tein, low fat diet supplemented with vitamin $\mathrm{B}, \mathrm{C}, \mathrm{K}$ and folic acid [1]. Micro nutrients have protective role against the oxidative stress either by scavenging free radicals or by their role in synthesizing or recycling of GSH, the main intracellular antioxidant molecule [34]. They also reported that Zinc protects against oxidative stress-related liver damage, despite not having direct antioxidant properties and iron can enhance ALD.

\section{DRUG THERAPY}

Complementary and alternative medicine agents such as zinc, milk thistle, SAMe have great therapeutic effect on ALD [3]. Corticosteroids are effective in patients with alcoholic hepatitis and treatment with pentoxyfylline appears to be a promising anti-inflammatory therapy $[3,4]$. Improving the SAMe to SAH ratio and removal of intracellular SAH can be potential therapeutic options in ALD [20]. Similarly use of TNF- $\alpha$ neutralizing agent (anti-TNF antibody) could be useful as a treatment strategy of ALD [4, 34]. Once cirrhosis has developed, it persists despite the cessation of alcohol use [21].

Recently, it has been found that chlormethiazole, an inhibitor of CYP2E1, blocked ethanol-induced steatosis by lowering macrovesicular fat accumulation, inhibiting oxidative stress, and up-regulating PPAR $\alpha$ protein levels in ethanol fed wild type mice [19]. It is reported that adenosyl-Lmethionine replenishes glutathione and attenuates mitochondrial lesions. He also reported that polyenylphosphatidylcholine fully prevents ethanol-induced septal fibrosis and cirrhosis [13]. Acetaldehyde-enhanced production of $\mathrm{H}_{2} \mathrm{O}_{2}$ and $\mathrm{O}_{2}{ }^{-}$ was decreased by the inhibitory effect of raloxifene on NADPH oxidase; and increasing intracellular GSH by Nacetylcysteine [17].

\section{TREATMENT WITH ANTIOXIDANT}

Treatment with various antioxidants decreased alcoholic liver damage in rats [21]. It has been found that grape leaf extract possesses antioxidant activity of polyphenolic compounds that reduced the activities of liver marker enzymes (AST, ALT, GGT), levels of lipid peroxidation markers (lipid hydroperoxide, TBARS), and restored the levels of nonenzymatic antioxidants (vitamin $\mathrm{E}$, vitamin $\mathrm{C}$ and reduced glutathione; GSH) and enzymatic antioxidants [catalase
(CAT), glutathione peroxidase (GPx), SOD and glutathioneS-transferase (GST)] in rats fed with ethanol and grape leaf extract [26]. So it is an effective antioxidant in alcohol induced oxidative damage.

Vitamin C, a natural antioxidant, with many cellular activities can protect the cells against alcohol-induced peroxidation [35]. They found that serum level of IL-6 and TNF- $\alpha$ were lower in rats treated with ethanol and ascorbic acid than in rats treated only with ethanol [35]. It has been found that lecithin, lecithin with vitamin B-complex and tocopheryl acetate (vitamin E) treatment significantly reduced AST, ALT, IL-10, $\gamma$ IFN activities and TBARS content, while significantly elevated GSH content and SOD activity compared to ethanol treated group of rats, indicating the promising therapeutic approach in controlling ethanol-induced immunomodulatory activities involving liver damage processes [29]. However, since the same cytokines are also necessary for the regeneration of tissue after the liver has been injured, cytokine antagonism is not without risk in patients with liver disease, and complete inhibition of TNF- $\alpha$ activity might impair hepatic recovery [21].

\section{DISCUSSION}

This study provides an in-depth analysis of mechanisms of ALD development and progression. ALD, usually characterized with AST/ALT ratio $>1$, can progress to cirrhosis overtime in some individuals but not in all [42]. Ingestion of high amount of alcohol induces intestinal damage which leads to significant increase in LPS permeability thereby contributing to inflammatory responses in ALD via kupffer cell activation [36]. Obesity also increases susceptibility to endotoxin-mediated liver injury and, thus, identifies the common mechanism for both alcohol and obesity-related liver diseases [43]. Abnormal methionine metabolism in liver and adipose tissue is an acquired metabolic abnormality in ALD which contribute to hyperhomocysteinemia, increased SAH levels, decreased adiponectin production, and reduce the ability to replete GSH $[9,14,34]$. GSH protects against $\mathrm{H}_{2} \mathrm{O}_{2}$ and $\mathrm{O}_{2}^{-}$injury in liver [17]. Rat model of ALD showed that acetaldehyde concentrates after the ingestion of alcoholic beverages which increases the formation of $\mathrm{H}_{2} \mathrm{O}_{2}$ and $\mathrm{O}_{2}{ }^{-}$in rat hepatic stellate cells [17]. 
A link was indicated between the induction of CYP2E1 by ethanol, the formation of hydroxyethyl free radicals and the stimulation of lipid peroxidation [28]. It has been demonstrated that the role of CYP2E1 in the pathogenesis of ALD in an animal model experiment where administration of CYP2E1 to the knockout mice restored steatosis and use of chlormethiazole to inhibit CYP2E1, blocked ethanol-induced steatosis in wild-type mice [19]. TNF- $\alpha$ is a proinflammatory cytokine that can trigger distinct signal pathways in liver cells through TNF- $\alpha$ receptor 1 via adapter molecules, including the intracellular cascades leading to apoptosis, as well as NF-kB and Jun kinase activation [20].

Generally, females are more susceptible to ALD indicating gender specific mechanisms e.g. sex hormones and upregulation and downregulation of many genes in female liver, making them more susceptible to alcohol consumption [39]. It is suggested that carriers of the T allele of the CD14 receptor gene have a 3- to 4- fold risk of developing advanced alcoholic liver disease [37]. Dietary model of steatohepatitis has proved that accumulation of FFA occurs despite substantial suppression of lipogenesis (reduced nuclear levels of lipogenic transcription factor, SREBP) and induction of triglyceride synthesis genes (acetyl CoA carboxylase and fatty acid synthase) [44]. Accumulation of FFA supports a lipotoxicity mechanism for liver injury [44]. Poor dietary habits also may have contributed to oxidative stress in the patients because they consumed lower amounts of antioxidant vitamins compared to control subjects [41].

Kupffer cells are resident macrophages of the liver and play an important role in its normal physiology and homeostasis as well as participating in the acute and chronic responses of the liver to toxic compounds [45]. Altered production of inflammatory mediators by kupffer cell [24] and increased hepatocyte sensitivity to TNF $\alpha$ were the two underlying mechanisms for the increased hepatotoxicity [43]. The abnormalities of the hepatic innate immune system (macrophages, neutrophils, natural killer T cells) lead to the increased production of inflammatory cytokines which contribute chronic inflammatory state of liver injury [24]. Treatment with various antioxidants has decreased the injury due to alcohol in different animal studies [21]. Anti-TNF and other anti-inflammatory drugs; and TNF-neutralizing agents are also being a potentially beneficial treatment in animal studies and clinical trials $[13,17,21]$.

\section{CONCLUSIONS}

ALD continues to be a major health problem with respect to both morbidity and mortality in many countries. Clinical studies have established a spectrum of ALD from simple steatosis to steatohepatitis, fibrosis, cirrhosis, hepatocellular carcinoma and liver failure. But bland steatosis, sometimes, remains stable for a number of years and will probably never progress in many cases. Excess production of cytokines and ROS; and shortage of GSH, and adiponectin production play key roles in the pathogenesis of liver injury. The oxidative damage induced by ethanol contributes in many ways to the pathogenesis of alcohol hepatotoxicity. The immune system becomes defective and anti-inflammatory cytokines and antioxidants are unable to overcome inflammation and fibrosis in progressive liver injury. Immunological reactions against antigens originating from hydroxyethyl free radicals and lipid peroxidation products can be regarded as an additional mechanism in the pathogenesis of alcoholic liver injury, as they contribute to chronic inflammatory process. Antioxidants and anticytokines related specific therapies are being practiced for the treatment of ALD with complete alcohol withdrawal. More studies are needed to clarify the specific effects of different diets and dietary components in the health of ALD patients. Additional research needs to be done to control and effectively manage ALD.

\section{CONFLICT OF INTEREST}

The author(s) confirm that this article content has no conflicts of interest.

\section{ACKNOWLEDGEMENTS}

None declared.

\section{REFERENCES}

[1] Zeng MD, Li YM, Chen CW, et al. Guidelines for the diagnosis and treatment of alcoholic liver disease. J Dig Dis 2008; 9: 113-6.

[2] Albano E. Oxidative mechanisms in the pathogenesis of alcoholic liver disease. Mol Aspects Med 2008; 29: 9-16.

[3] Brave A, Khan R, Marsano L, Ravindra KV, McClain C. Treatment of alcoholic liver disease. Ann Hepatol 2008; 7: 5-15.

[4] Bergheim I, McClain CJ, Arteel GE. Treatment of alcoholic liver disease. Dig Dis 2005; 23: 275-84.

[5] Mueller S, Millonig G, Sarovska L, et al. Increased liver stiffness in alcoholic liver disease: Differentiating fibrosis from steatohepatitis. World J Gastroenterol 2010; 16: 966-72.

[6] Amarapurkar A, Ghansar T. Fatty liver: experience from western India. Ann Hepatol 2007; 6: 37-40.

[7] Vidali M, Stewartz SF, Albano E. Interplay between oxidative stress and immunity in the progression of alcohol-mediated liver injury. Trends Mol Med 2008; 14: 63-71.

[8] Adachi M, Brenner DA. Clinical syndromes of alcoholic liver disease. Dig Dis 2005; 23: 255-63.

[9] Song Z, Zhou Z, Deaciuc I, Chen T, McClain CJ. Inhibition of adiponectin production by homocysteine: a potential mechanism for alcoholic liver disease. Hepatology 2008; 47: 867-79.

[10] Denk H, Stumptner C, Fuchsbichler A, Zatloukal K. Alcoholic and non-alcholic steatohepatitis. Verh Dtsch Ges Pathol 2005; 89: 13743.

[11] Tabassum F, Khurshid R, Karim S, Akhtar MS. Metabolic Effects of alcoholism and its relationship with alcoholic liver disease. J Ayub Med Coll Abbottabad 2001; 13: 19-21.

[12] American Liver Foundation. Alcohol-related liver disease. [Cited: $12^{\text {th }}$ May 2012]; Available from: http://www.liverfoundation.org/ abouttheliver/info/alcohol/.

[13] Lieber CS. Ethanol metabolism, cirrhosis and alcoholism. Clin Chim Acta 1997; 257: 59-84.

[14] Lumeng L, Crabb DW. Alcoholic liver disease. Curr Opin Gastroenterol 2001; 17: 211-20.

[15] Hasse JM, Matarese LE. Medical nutrition therapy for liver, biliary system, and exocrine pancreas disorders. In: Mahan LK, EscottStump S, Eds. Krause's food, nutrition and diet therapy. $11^{\text {th }}$ ed. Philadelphia: Elsevier 2004; pp. 738-67.

[16] De Minicis S, Brenner DA. Oxidative stress in alcoholic liver disease: role of NADPH oxidase complex. J Gastroenterol Hepatol 2006; 23: s98-103.

[17] Novitskiy G, Traore K, Wang L, Trush MA, Mezey E. Effects of ethanol and acetaldehyde on reactive oxygen species production in rat hepatic stellate cells. Alcohol Clin Exp Res 2006; 30: 1429-35.

[18] Lu Y, Cederbaum AI. CYP2E1 and oxidative liver injury by alcohol. Free Radic Biol Med 2008; 44: 723-38.

[19] Lu Y, Zhuge J, Wang X, Bai J, Cederbaum AI. Cytochrome P450 $2 \mathrm{E} 1$ contributes to ethanol-induced fatty liver in mice. Hepatology 2008; 47: 1483-94. 
[20] Song Z, Zhou Z, Uriarte S, et al. S-Adenosylhomosysteine sensitizes to TNF- $\alpha$ hepatotoxicity in mice and liver cells: a possible etiological factor in alcoholic liver disease. Hepatology 2004; 40: 989-97.

[21] Tilg H, Diehl AM. Cytokines in alcoholic and non alcoholic steatohepatitis. N Engl J Med 2000; 343: 1467-76.

[22] Miller AM, Wang H, Bertola A, et al. Inflammation-associated interleukin-6/signal transducer and activator of transcription 3 activation ameliorates alcoholic and nonalcoholic fatty liver diseases in interleukin-10-deficient mice. Hepatology 2011; 54: 846-56.

[23] $\mathrm{Ki} \mathrm{SH}$, Park $\mathrm{O}$, Zheng $\mathrm{M}$, et al. Interleukin-22 treatment ameliorates alcoholic liver injury in a murine model of chronicbinge ethanol feeding: Role of STAT3. Hepatology 2010; 52: 1291-300.

[24] Yang L, Diehl AM. Role of immune response in nonalcoholic fatty liver disease: evidence in human and animal studies. In: Gershwin ME, Vierling JM, Manns MP, Eds. Liver immunology: principles and practice. Totowa: Humana Press 2007; pp. 337-45.

[25] Reuben A. Alcohol and the liver. Curr Opin Gastroenterol 2008; 24: 328-38.

[26] Pari L, Suresh A. Effect of grape (Vitis vinifera L.) leaf extract on alcohol induced oxidative stress in rats. Food Chem Toxicol 2008; 46: $1627-34$.

[27] Day CP, James OF. Steatohepatitis: a tale of two "hits"? Gastroenterology 1998; 114: 842-5.

[28] Albano E, Clot P, Morimoto M, Tomasi A, Ingelman-Sundberg M, French SW. Role of cytochrome P4502E1-dependent formation of hydroxyethyl free radical in the development of liver damage in rats intragastrically fed with ethanol. Hepatology 1996; 23: 155-63.

[29] Das SK, Gupta G, Rao DN, Vasudevan DM. Effect of lecithin with vitamin B-complex and tocopheryl acetate on long term effect of ethanol induced immunomodulatory activities. Indian J Exp Biol 2007; 45: 683-8.

[30] Albano E. Alcohol, oxidative stress and free radical damage. Proc Nutr Soc 2006; 65: 278-90.

[31] Bailey MS, Cunningham CC. Contribution of mitochondria to oxidative stress associated with alcoholic liver disease. Free Radic Biol Med 2002; 32: 11-6.

[32] Cave M, Deaciuc I, Mendez C, et al. Nonalcoholic fatty liver disease: predisposing factors and the role of nutrition. J Nutr Biochem 2007; 18: 184-95.
[33] Lu SC, Huang Z, Yang H, Mato JM, Avila MA, Tsukamoto H. Changes in methionine adenosyltransferase and S-adenosylmethionine homeostasis in alcoholic rat liver. Am J Physiol Gastrointest Liver Physiol 2000: G178-85.

[34] Everitt H, Patel VB, Tewfik I. Nutrition and alcoholic liver disease. Nutr Bull 2007; 32: 138-44.

[35] El-Taukhy MA, Salama SM, Abou-Shousha SA, Ismail SS, Saleh M. Effects of chronic ethanol and vitamin $\mathrm{C}$ administration on production of tumor necrosis factor-alfa and interleukin- 6 in rats Egypt J Immunol 2006; 13: 1-10.

[36] Parlesak A, Schafer C, Schutz T, Bode JC. Increased intestinal permeability to macromolecules and endotoxemia in patients with chronic alcohol abuse in different stages of alcohol-induced liver disease. J Hepatol 2000; 32: 742-7.

[37] Jarvelainen HA, Prpana A, Perola M, Savolainen VI, Karhunen PJ, Lindros KO. Promotor polymorphism of the CD14 endotoxin receptor gene as a risk factor for alcoholic liver disease. Hepatology 2001; 33: 1148-53.

[38] Duryee MJ, Klassen LW, Thiele GM. Immunological response in alcoholic liver disease. World J Gastroenterol 2007; 13: 1938-46.

[39] Sharma MR, Polavarapu R, Roseman D, et al. Increased severity of alcoholic liver injury in female verses male rats: a microarray analysis. Exp Mol Pathol 2008; 84: 46-58.

[40] Seitz HK, Stickel F. Alcoholic liver disease in the elderly. Clin Geriatr Med 2007; 23: 905-21.

[41] Musso G, Gambino R, De Michieli F, et al. Nitrosative stress predicts the presence and severity of nonalcoholic fatty liver at different stages of the development of insulin resistance and metabolic syndrome: possible role of vitamin A intake. Am J Clin Nutr 2007; 86: 661-71.

[42] Kazemi-Shirazi L, Veloso MP, Frommlet F. Differentiation of nonalcoholic from alcoholic steatohepatitis: are routine laboratory markers useful? Wien Klin Wochenschr 2008; 120: 25-30.

[43] Yang SQ, Lin HZ, Lane MD, Clemens M, Diehl AM. Obesity increases sensitivity to endotoxin liver injury: Implications for the pathogenesis of steatohepatitis. Proc Natl Acad Sci USA 1997; 94: 2557-62.

[44] Larter CZ, Yeh MM, Haigh WG, et al. Hepatic free fatty acids accumulate in experimental steatohepatitis: role of adaptive pathways. J Hepatol 2008; 48: 638-47.

[45] Jaeschke H, Farhood A. Neutrophil and kupffer cell-induced oxidant stress and ischemia-reperfusion injury in rat liver. Am J Physiol Gastrointest Liver Physiol 1991; 260: 355-62.

Received: April 30, 2012

Revised: May 13, 2012

Accepted: June 02, 2012

(C) Adhikari and Tirosh; Licensee Bentham Open.

This is an open access article licensed under the terms of the Creative Commons Attribution Non-Commercial License (http://creativecommons.org/licenses/ by-nc/3.0/) which permits unrestricted, non-commercial use, distribution and reproduction in any medium, provided the work is properly cited. 